\title{
History-Dependence of Muscle Excitation and Oxygenation During Isometric Knee Extension Force Production
}

\author{
Junkyung Song ${ }^{1}$ MS, Kitae Kim ${ }^{1}$ PhD, Sungjun Lee ${ }^{1}$ MS, Jiseop Lee ${ }^{1}$ MS, Jaebum Park ${ }^{1,2}$ PhD \\ 'Department of Physical Education, Seoul National University, Seoul; nstitute of Sport Science, Seoul National University, Seoul, Korea
}

PURPOSE: This study examined the issue of history-dependence on muscle excitation and oxygenation by using surface electromyography (EMG) and near-infrared spectroscopy (NIRS).

METHODS: Eight male participants performed isometric knee extension force production tasks that started at four levels of initial force production (30, 40, 60, 70\% of maximal voluntary contraction, MVC) and commonly converged to the production of $50 \%$ of MVC by increasing or decreasing knee extension forces. We quantified the integrated electromyogram (iEMG), total hemoglobin (tHb), and the desaturation rate of muscle tissue (TSI SLOPE$_{\text {) }}$ of the vastus lateralis and compared the differences in the quantified variables between the experimental conditions.

RESULTS: For the four levels of initial forces production, all the variables showed significant linear relationships with outcome forces. However, the magnitudes of the variables were varied depending on the contraction history at the terminal phase where the identical level of force production ( $50 \%$ of MVC) was required. At the terminal phase, the iEMG was affected by the time history of both mode (e.g., increment or decrement) and magnitude (e.g., $10 \%$ or $20 \%$ change of MVC), while the tHb and TSIsLope showed significant differences between the time history of mode only.

CONCLUSIONS: The main result of the current study demonstrates the history-dependence of the changes in physiological demands in muscle activation. In particular, the indices of muscle excitation and oxygenation at the same level of force were varied depending on the time history of contraction, which implies the indices of muscle excitation and oxygenation may not be invariant component to specify the levels of outcome forces.

Key words: Muscular history-dependence, Hysteresis, Surface electromyography, Near-infrared spectroscopy

\section{INTRODUCTION}

Over the years, the mechanisms of the change in physiological variables and their mechanical consequences have been extensively studied. The mechanical outcome forces demonstrate the proportional relationship with the parameters of the electromyography (EMG) and Near-infrared spectroscopy (NIRS) [1-3]. The NIRS is a non-invasive technique that has been applied to quantify the local oxidative metabolism of the tissues (e.g., muscle) during exercise. In particular, the NIRS measures the total hemoglobin $(\mathrm{tHb})$ and tissue oxygen saturation index (TSI) during voluntary activities, which are indicative of regional blood vol- ume [4] and dynamic balance between oxygen supply and consumption [5], respectively.

Skeletal muscles are physiological structure whose primary function is to convert chemical and electrical resources to the mechanical outcome. The changes in force demand, therefore, yield the adjusted levels of physiological parameters of muscle activation [6]. The process of muscle activation requires metabolic energy, and the mechanism of oxidative phosphorylation is responsible for the generation of the muscular energy source for operating the cross-bridge cycle $[7,8]$. The EMG amplitude and patterns were linearly related to the steady-state levels of outcome forces during isometric contraction [9-11], and the studies that employed

Corresponding author: Jaebum Park Tel +82-2-880-7620 Fax+82-2-872-2867 E-mail parkpe95वsnu.ac.kr

Keywords Muscular history-dependence, Hysteresis, Surface electromyography, Near-infrared spectroscopy

Received 4 Sep 2020 Revised 7 Oct 2020 Accepted 12 Oct 2020

() This is an Open Access article distributed under the terms of the Creative Commons Attribution Non-Commercial License (https://creativecommons.org/licenses/by-nc/4.0/) which permits unrestricted non-commercial use, distribution, and reproduction in any medium, provided the original work is properly cited. 
NIRS also provided evidence that the mechanical outcome forces show a close-to-linear relationship with the parameters of muscle oxygenation and hemodynamics $[3,4,12,13]$.

Unlike the proportional relationship between the action potential and outcome force, blood flow to the muscle is reduced or nearly constant at higher levels of muscle contraction due to the unchanged intramuscular pressure (IMP) $[13,14]$. In fact, it is well known that skeletal muscles have a nonlinear force-length relationship depending on its history of contraction, which is referred to as the muscular hysteresis [15-17]. We assume that considerable discrepancy is partially due to a variety of history-dependent effects on the outcomes of the neuromuscular and motor systems since the contractile properties of the muscles depend on the history of activation of the motor neurons [18-20]. Hence, the aforementioned factors regarding the mechanical properties and the neural aspects of muscle activation are possibly affected by muscle contraction history.

To the best of our knowledge, few studies have focused on the issue of a history-dependent relation between mechanical outcome forces (or torques) and physiological parameters or further speculation on its mechanism. The quantifications of the history-dependent physiological demands would provide better insight into the mechanisms of voluntary muscle contraction. Therefore, the current experimental purpose was to examine the issue of history-dependence on muscle excitation and oxygenation using surface EMG (sEMG) and NIRS. We formulated two hypotheses: 1) The sEMG and NIRS variables will change proportionally with the magnitudes of outcome force when different levels of force magnitude are reached from resting status (i.e., zero force). 2) The chang- es of EMG and NIRS variables at the same outcome force will be varied according to the history of contraction modalities (i.e., increase or decrease force) and the magnitudes of difference in levels from initial to terminal force levels.

\section{METHODS}

\section{Participants}

Eight healthy male volunteers participated in the current experiment. Their average age, weight, height were $29.88 \pm 4.55$ years, $74.38 \pm 3.02 \mathrm{~kg}$, and $173.88 \pm 2.79 \mathrm{~cm}($ mean $\pm S D)$, respectively. The dominant side of the leg was the right for all participants. No participant had a previous history of neuropathies or traumas to their lower extremities, and all the participants did not have a cigarette smoking for more than six months. The experimental procedure of the study was performed in accordance with the recommendations of the Seoul National University Institutional Review Board (IRB No. 1712/001-006).

\section{Apparatus}

A customized steel frame was designed to perform unilateral isometric knee extension tasks at a specific knee joint angle (Fig. 1A). A single mid-size force transducer (MC3A, AMTI, Watertown, MA USA; 76×76 $\times 76.2 \mathrm{~mm}$ ) was attached to the steel frame to measure the isometric knee extension force, and the force transducer was firmly affixed to the steel frame near the ankle joint along with the frame (Fig. 1A). The orientation of the force transducer was aligned in such a way that the $\mathrm{z}$-axis force component was perpendicular to the long axis of the shank. The

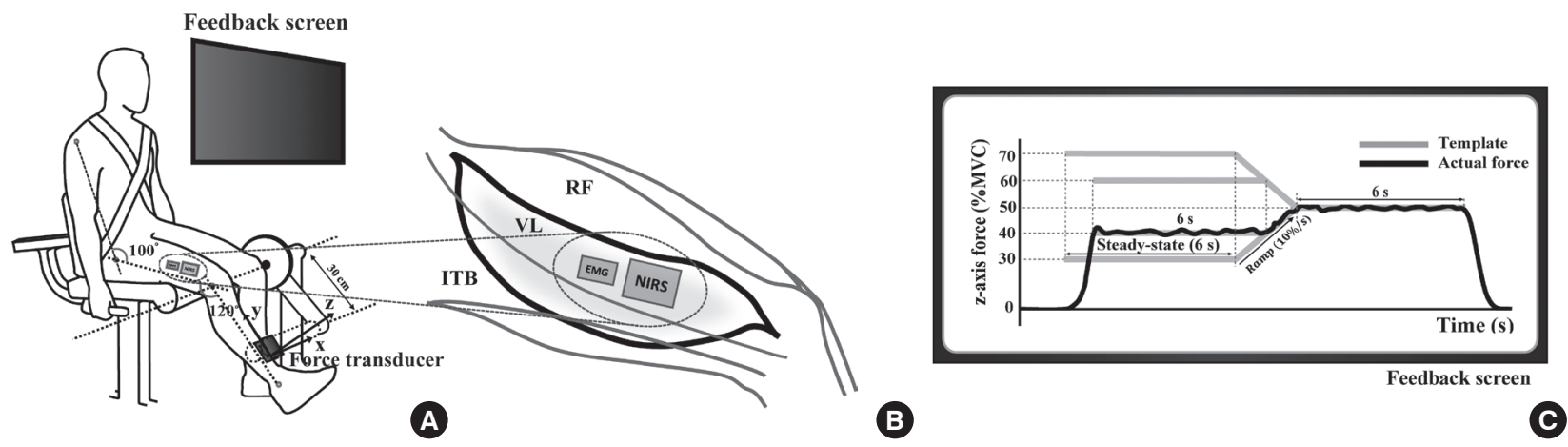

Fig. 1. (A) Experimental setup and visual feedback screen used in the force tracking and magnitude transition task. The force transducer was mounted with the z-axis perpendicular to the long axis of the shank. (B) The surface EMG and portable NIRS were attached to the belly of the vastus lateralis muscle. (C) The computer screen for providing the real-time visual feedback of the knee extension force. The template on the screen provided magnitudes of target forces. The force tracking tasks started steady force production at four force levels (initial phase: 30, 40, 60, 70\% of MVC) and commonly converged to $50 \%$ of MVC (terminal phase) by increasing or decreasing forces. 
wireless sEMG (Trigno Wireless EMG System, Delsys, Natick, MA, USA) electrode was attached to the belly of the VL muscle of the right leg (Fig. 1B). In addition, the portable NIRS device (PortaMon, Artinis Medical Systems, Elst, NL) was attached adjacent to the EMG electrode (Fig. 1B) on the same muscle. All three measurement systems were physically synchronized, and the force transducer, EMG, and NIRS were all sampled at frequencies of 200,2,000, and $10 \mathrm{~Hz}$, respectively. In the current experiment, we measured the EMG and NIRS data of the VF exclusively because the VL has been known to have the most salient linearity of outcome forces among the quadriceps femoris [21]. Further, the VL is considered a mono-articular muscle that mainly works for the force generation, not for regulating joint moments [22].

\section{Experimental procedure}

The participant sat in a steel frame chair facing a 20-inch computer screen at eye level position for providing the real-time force feedback. Note that the force about the $\mathrm{z}$-axis component was used and displayed exclusively for the current experiment. The distal part of the shank was secured to the force transducer using a Velcro strap to prevent the thigh and shank movement (Fig. 1A). The hip and knee joint angles with respect to the horizontal axis were fixed at $100^{\circ}$ and $120^{\circ}$, respectively $[23,24]$. A strap across the waist and trunk prevented excessive movement, and the participants were required to hold the side grips tightly by using both hands (Fig. 1A).

The experiment consisted of two blocks. The first task was the maximal voluntary contraction (MVC) force production task. The participants were instructed to increase knee extension force gradually from the resting level and to reach a maximal level within 5 second. Each participant performed three consecutive attempts, and the highest force value was used for the determination of the target force values for the next tasks. The EMG signal of the VL muscle was also measured during the MVC task to normalize EMG signals for further measurement. The second task was the force tracking and magnitude transition task. A single trial lasted 20 second consisting of 6 second of the initial steady-state phase followed by the ramp-up ( $\left.F_{U P}\right)$ or ramp-down phase ( $\left.F_{\text {DOwN }}\right)$, and another 6 second of the terminal steady-state phase. The constrained force template was provided on the computer screen (Fig. 1C). There were four conditions of the initial force levels including 30, 40, 60, and $70 \%$ of $\mathrm{MVC}$ (i.e., $\mathrm{MVC}_{30}, \mathrm{MVC}_{40}, \mathrm{MVC}_{60}$, and $\mathrm{MVC}_{70}$ ), and the terminal level of force was the same for all conditions with setting at $50 \%$ of $\mathrm{MVC}\left(\mathrm{MVC}_{50}\right)$. The rate of force change was set at $\pm 10 \%$ of $\mathrm{MVC} / \mathrm{s}$, which was intended to guide smooth transitions of force magnitude. The absolute difference of force magnitude from the initiation to termination phase for the $\mathrm{MVC}_{30}$ and $\mathrm{MVC}_{70}$ was $20 \%$ of $\mathrm{MVC}\left(\Delta \mathrm{F}_{20}\right)$ while being $10 \%$ of $\mathrm{MVC}\left(\Delta \mathrm{F}_{10}\right)$ for the $\mathrm{MVC}_{40}$ and $\mathrm{MVC}_{60}$ conditions. Each participant performed 5 trials for each condition; thus, a total of 20 trials (5 trials $\times 4$ conditions) was performed in randomized order. A 2 minutes rest was allowed after the completion of each trial, and an extra 5 minutes rest was allowed after the completion of five trials. No participant reported muscle fatigue during the experiment.

\section{Data Analysis}

The force data were digitally filtered using a zero-lag fourth-order lowpass Butterworth filter with a $10 \mathrm{~Hz}$ cut-off. The steady-state periods for both initial and terminal phases over 5 second were detected where the stable level of force was observed in the middle of the period. The first and the last 0.5 second within each trial were excluded from the analysis to avoid edge effect. The identified steady-state time information was used in common to analyze the EMG and NIRS data of the corresponding trial.

Root-mean-squared error (RMSE) as an index of performance accuracy of the force production was computed for a particular trial during the initial and terminal steady-state phases, separately. Further, the RMSE values were normalized by the values of the template forces (RM$\mathrm{SE}_{\mathrm{NORM}}$ ) of the corresponding condition.

The integrated EMGs (iEMGs) over the two steady-state phases for individual trials were computed. The procedure of the EMG data processing was as follows: 1) the application of a notch-filter at $60 \mathrm{~Hz}$ and 2) 10 to $450 \mathrm{~Hz}$ band-pass \& second-order zero-lag Butterworth filters. 3) Fullwave rectification followed by applying a $100 \mathrm{~ms}$ moving-average window for detecting EMG envelopes. 4) 1)-2) steps were done for the EMG signals from the MVC trials $\left(\mathrm{EMG}_{\mathrm{MVC}}\right)$, and the $\mathrm{EMG}_{\mathrm{MVC}}$ was integrated ( $i \mathrm{EMG}_{\mathrm{MVC}}$ ) over the same time window (i.e., $100 \mathrm{~ms}$ ) where the peak knee extension force was observed. 5) The processed EMG signals were normalized by $i \mathrm{EMG}_{\mathrm{MVC}}$ and integrated over the periods of the steadystate phases to determine the normalized $i \mathrm{EMG}\left(i \mathrm{EMG}_{\mathrm{NORM}}\right)$. In addition, we calculated the mean power frequencies (MPF) of the EMG signals at the terminal steady-state phase for each of the randomized 20 trials. Note that a significant decrease in MPF in the spectral domain of the EMG signal possibly indicates a decrease in muscle fiber propagation velocity due to muscle fatigue [25].

The modified Beer-Lambert law and spatially resolved spectroscopy 
(SRS) methods were used to calculate the two variables, including the total hemoglobin $(\mathrm{tHb})$ by relative estimates of oxy-and deoxy-hemoglobin and the tissue oxygen saturation index (TSI) from the NIRS measurement [26]. The baseline values of the TSI and $\mathrm{tHb}$ were determined as the average values over 30 second before the onset of muscle contraction. The baseline value was subtracted from the TSI and $\mathrm{tHb}$ value during a particular trial [27]. Further, average values of tHb over 5 second at each of the initial and terminal steady-state periods were computed. The TSI desaturation slope $\left(\mathrm{TSI}_{\mathrm{SLOPE}}\right)$ as an index of muscle oxygen demand was computed as a negative slope of the least-squared regression line of the TSI values during the sustained muscle contraction over 5 second [28]. Note that the larger TSISLOPE represents a greater muscle oxygen demand that is associated with a higher energy consumption [4]. Subsequently, the average values of the $i \mathrm{EMG}_{\mathrm{NORM}}, \mathrm{tHb}$, and TSISLOPE across five trials for each condition and participant were used for further statistical analysis.

\section{Statistical Analysis}

Prior to the main statistical analysis, we performed two preliminary statistical tests to examine the effect of repetitive trials on muscle fatigue and performance accuracy using the MPF and RMSE $E_{\text {NORM }}$ data, respectively. For the $i \mathrm{EMG}_{\mathrm{NORM}}$ and NIRS indices (e.g., tHb, TSI $\mathrm{SLOPE}_{\text {) }}$ for the four levels of the initial force magnitudes, separate linear regressions analyses were performed to examine the relations of the force magnitudes with $i \mathrm{EMG}_{\mathrm{NORM}}$ and NIRS indices, respectively (Fig. 2). Likewise, the relations between $i \mathrm{EMG}_{\mathrm{NORM}}$ and NIRS indices in the initial and terminal phases were examined using separated linear regression analyses (Fig. 4 and 5). If the regression lines showed significant relationships, we further tested whether the outcomes of the regression parameters (i.e., $r$ values) were significantly different [29]. For the comparisons of $i \mathrm{EMG}_{\mathrm{NORM}}$ and NIRS indices at the terminal steady-state phase, ANOVAs with repeated-measures were used with factors Mode (two levels: $\mathrm{F}_{\mathrm{UP}}$ and $\mathrm{F}_{\mathrm{DOwN}}$ ) and Magnitude (two levels: $\Delta \mathrm{F}_{10}$ and $\Delta \mathrm{F}_{20}$ ). The indices of post calculation of the effect size (partial eta-squared, $\eta p^{2}$ ) for every comparison were presented. We conducted all statistical analyses using SPSS 24.0 (IBM, Armonk, NY USA), and the level of significance was set at $p<.05$.
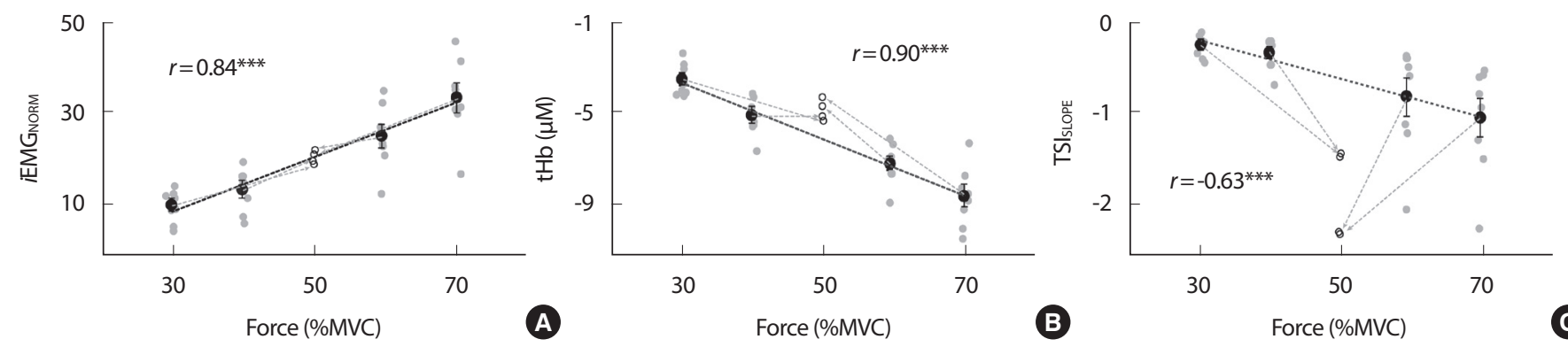

Fig. 2. The relationship between four levels of forces in the initial steady-state phase (30, 40, 60, and 70\% of MVC) and (A) iEMG NORM, $_{\text {, }}(B)$ tHb, and (C) TSIsLOPE. Small grey circles represent the data from individual participants ( $n=8$ with four conditions), and the average data across the participants are shown with black circles and standard error bars. Black dashed lines indicate linear regression lines, and the coefficients of correlation are shown in each panel $\left({ }^{* * *} p<.001\right)$. Four Small open circles in each panel represent the terminal steady-state values at $50 \%$ of MVC with grey dashed lines linking to the initial steady-state values.

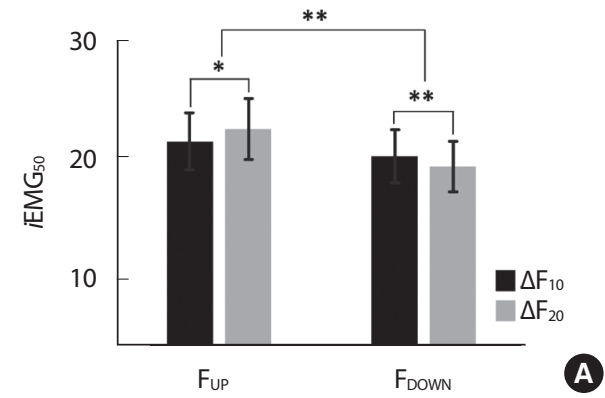

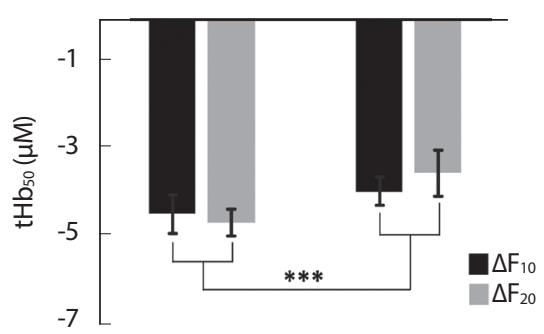

FuP
B

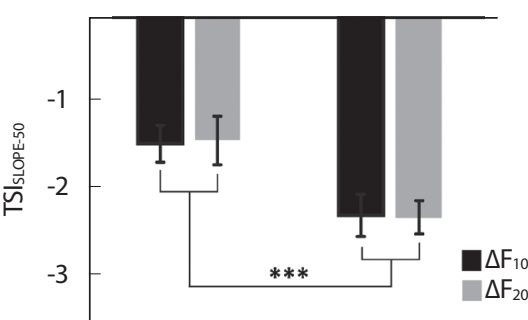

Fup

Fig. 3. (A) iEMG $\mathrm{EMRM}_{\text {, }}(\mathrm{B}) \mathrm{tHb}$, and (C) TSIsLope at the terminal steady-state values ( $50 \%$ of $\mathrm{MVC}$ ) for $\Delta \mathrm{F}_{10}$ and $\Delta \mathrm{F}_{20}$ during Fup and $\mathrm{F}_{\text {Down }}$ modes. Average values across participants are presented with bars representing standard errors $\left({ }^{* * *} p<.001,{ }^{* *} p<.01,{ }^{*} p<.05\right)$. 


\section{RESULTS}

\section{Integrated EMG (iEMG NORM $_{\text {) }}$}

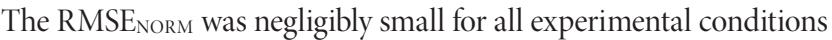
(on average, $1.64 \pm 0.51 \%$ ). Also, the RMSENorm between initial and terminal phase were not significantly different. The regression analysis confirmed that the MPF of the EMG signals had no significant changes over the repeated trials $(r=.04, p>.05)$.

The $i \mathrm{EMG}_{\mathrm{NORM}}$ increased with the magnitudes of knee extension forces at the initial phases, and a significant relationship between $i \mathrm{EMG}_{\mathrm{NORM}}$ and the forces was the best fit by a linear regression fitting $(r=.84, p<$ .001 , Fig. 2A). However, the comparisons of $i \mathrm{EMG}_{\text {NORM }}$ of the terminal phase between the experimental conditions showed a significant difference depending on the conditions. The $i \mathrm{EMG}_{\text {NORM }}$ was larger in the $\mathrm{F}_{\mathrm{UP}}$ mode than in the $\mathrm{F}_{\text {DOWN }}$ mode. During the $\mathrm{F}_{\mathrm{UP}}$ mode, $i \mathrm{EMG}_{\mathrm{NORM}}$ at the $\Delta \mathrm{F}_{20}$ magnitude was larger than at $\Delta \mathrm{F}_{10}$ magnitude. However, this pattern was reversed in the $\mathrm{F}_{\text {DOWN }}$ mode (at $\Delta \mathrm{F}_{10}>$ at $\Delta \mathrm{F}_{20}$, Fig. $3 \mathrm{~A}$ ). These findings were confirmed by a two-way repeated measures ANOVA on $i$ EMG $_{\text {NORM }}$ with factors Mode and Magnitude, which showed significant main effects of $\operatorname{Mode}\left(F_{[1,7]}=15.28, p=.006, \eta p^{2}=.70\right)$ with a significant Mode $\times$ Magnitude $\left(F_{[1,7]}=17.84, p=.004, \eta p^{2}=.72\right)$. The significant factor interaction reflected the fact that the $i \mathrm{EMG}_{\mathrm{NORM}}$ at $\Delta \mathrm{F}_{20}>$ at $\Delta \mathrm{F}_{10}$ in the Fup mode $(p=.01)$, while the $i \mathrm{EMG}_{\text {NORM }}$ at $\Delta \mathrm{F}_{10}>$ at $\Delta \mathrm{F}_{20}$ in the $\mathrm{F}_{\text {DOw }}$ mode $(p=.002)$.

\section{Total hemoglobin $(\mathrm{tH} b)$ \& rate changes of tissue oxygen saturation index (TSISLOPE)}

Both the $\mathrm{tHb}$ and TSIsLOPE decreased linearly with the magnitudes of outcome force during initial force production phases. The linear regression analysis showed significant fitting for both variables (tHb: $r=-.90$, $p<.001$; TSI $_{\text {SLOPE }}: r=-.63, p<.001$ ) (Fig. $2 \mathrm{~B}$ and C). During the terminal steady-state force production $\left(\mathrm{MVC}_{50}\right)$, the $\mathrm{tHb}$ was smaller in the $\mathrm{F}_{\mathrm{UP}}$ mode than in the $F_{D O W N}$ mode with no effect of the changes in force magnitudes Fig. 3B. Similarly, the TSIsLope also differed depending on the contraction mode, which showed a steeper decrease in the $\mathrm{F}_{\mathrm{DOWN}}$ mode than in the $\mathrm{F}_{\mathrm{UP}}$ mode (Fig. 3C). The two-way repeated measures
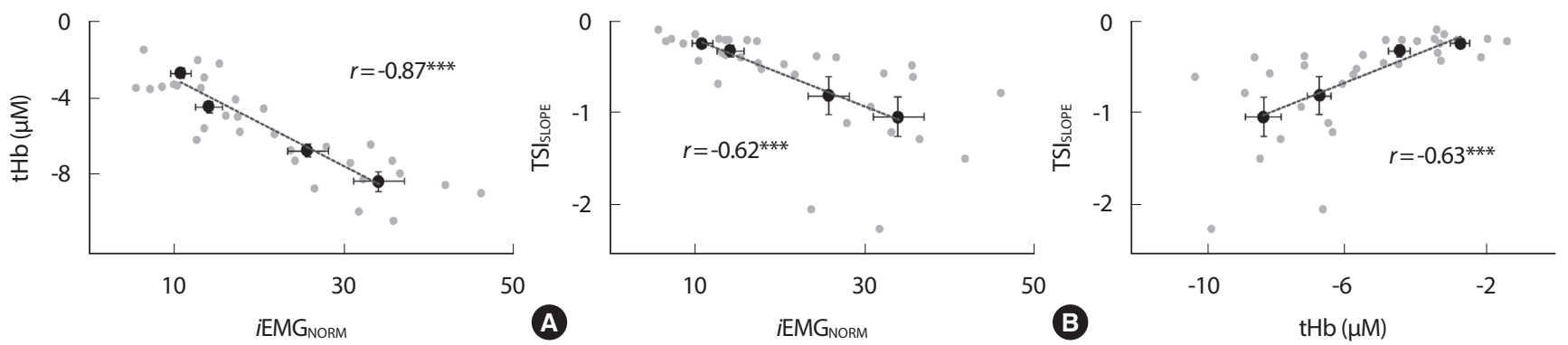

C

Fig. 4. The relationship between $i E M G_{\text {NORM }}$ and NIRS indices at the initial force production phase $\left(30,40,60\right.$, and $70 \%$ MVC). (A) $i E M G_{N O R M}$ Vs. tHb, (B) iEMG

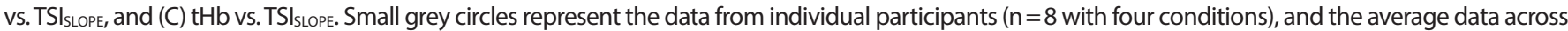
the participants are shown with black circles and standard error bars. Black dashed lines indicate linear regression lines, and the coefficients of correlation are shown in each panel $(* * *<.001)$.
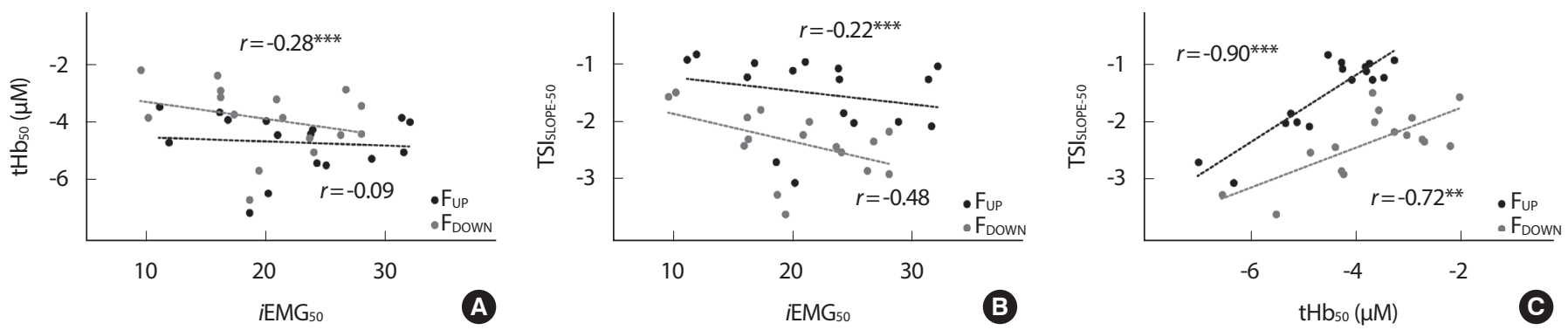

Fig. 5. The relationship between iEMGNorm and NIRS indices at the terminal force production phase (50\% of MVC). (A) iEMGNORM Vs. tHb, (B) iEMGNORM Vs. TSIs. LOPE, and (C) tHb vs. TSIsLope. Black and grey circles represent the data from individual participants ( $\mathrm{n}=8$ with four conditions) for the Fup and Fown mode, respectively. Black and grey dashed lines indicates linear regression lines for the Fup and Foown mode, respectively, and the coefficients of correlation are shown in each panel $\left({ }^{* * *} p<.001,{ }^{* *} p<.01\right)$. 
ANOVAs showed a significant main effect of Mode (tHb: $F_{[1,7]}=171.59$, $p<.001, \eta p^{2}=.96$; TSI $_{\text {SLOPE }}: F_{[1,7]}=86.19, p<.001, \eta p^{2}=.93$ ) without factor interaction

\section{Comparisons between iEMG and NIRS indices}

During the initial steady-state production, both the $\mathrm{tHb}$ and TSI $\mathrm{SLPPE}_{\mathrm{S}}$ decreased significantly with the $i$ EMGs ( $i \mathrm{EMG}_{\text {Norm }} \mathrm{Vs}$. $\mathrm{tHb}: r=-.87$, $p<.001, i$ EMG $_{\text {NORM V}}$ vs. TSISLOPE: $r=-.62, p<.001$ ), while there was a significant positive correlation between the $\mathrm{tHb}$ and TSI $\mathrm{SLPPE}_{\text {L }}(\mathrm{tHb}$ vs. TSIS LOPE: $r=.63, p<.001)$ as shown in Fig. 4 . We further tested the statistical difference on the correlation coefficients (i.e., $r$-values) and slopes of the regressions of between ' $i \mathrm{EMG}_{\text {NORM }}$ vs. tHb' and 'iEMG $\mathrm{EMR}_{\text {NOR V}}$ V. TSISLOPE'. The results showed that the $r$-value of the regression of ' $i E M G_{\text {NORM }}$ vs. $\mathrm{tHb}(r=-.87)$ ' was significantly smaller than that of ' $i \mathrm{EMG}_{\text {NORM }}$ Vs. TSIsLOPE $(r=-.62)$ ' $(p=.03)$. In other words, $i$ EMG $_{\text {NORM values had stronger }}$ linear relation to $\mathrm{tHb}$ than to TSIsLope. During the terminal steady-state force production, $i \mathrm{EMG}_{\text {NORM }}$ showed no significant relationship with both $\mathrm{tHb}$ and TSI SLOPE for both $\mathrm{F}_{\mathrm{UP}}$ and $\mathrm{F}_{\mathrm{DOWN}}$ modes (Fig. 5A and B). On the contrary, there was a significant positive correlation between the tHb and TSI ${ }_{\text {SLOPE }}$ for both $\mathrm{F}_{\mathrm{UP}}$ and $\mathrm{F}_{\mathrm{DOWN}}$ modes ( $\mathrm{F}_{\mathrm{UP}}: r=.90, p<.001$; Fown: $r=.72, p=.002$ ), while there were no statistical differences on $r$ values and slopes between $\mathrm{F}_{\mathrm{UP}}$ and $\mathrm{F}_{\mathrm{DOWN}}$ modes (Fig. 5C).

\section{DISCUSSION}

The current results support the two formulated hypotheses in the Introduction. We observed the linear changes of the $i$ EMG and the NIRS indices with respect to the force magnitudes, which supports the first hypothesis. We also observed non-monotonic changes in the $i$ EMG and NIRS indices depending on whether the same magnitudes of knee extension forces were achieved through smooth increasing or decreasing force magnitudes over time, which supports the second hypothesis.

It has been reported that the vastus lateralis $(\mathrm{VL})$ is the most reliable muscle by which to observe the linear relationship of force with the $i \mathrm{EMG}$ among the group of the quadriceps femoris muscles [21]. For these reasons, we selected the VL exclusively for the testing muscle among the quadriceps group. Our finding is also in line with this claim by showing a significant linear relationship between the $i \mathrm{EMG}$ of the VL muscle and knee extension forces when the steady-state knee extension forces were produced in the absence of a time-history of their magnitude changes. However, the proportional relationship was neither linear nor converged on the same values for the same outcome force if the same outcome forces had a different time-history as to the changes in force magnitude. Since the rate change of force magnitudes is a critical factor as to the resistance of deformation [30,31], we purposefully designed the experimental protocols such that the rate changes of force magnitude was constrained and the same for all experimental conditions. Also, it was confirmed that muscle fatigue and performance errors seem to have minimal effect on the history effect on other variables. Therefore, the historyeffect of the current physiological variables describes that the direction of changes in outcome force by the muscle (i.e., changes in force magnitudes) could be a key factor of behavior regarding the non-linearity of a physiological process in the current experiment. It has been known that the rate of the firing frequencies of the motor units at the same force level is lower during muscle relaxation (i.e., decreasing force) than during contraction (i.e., increasing force) [32-34]. By combining the existing knowledge and the current results, these mechanical properties of muscles allow for more efficient tension development during decreasing force than increasing force. Thus, the lower muscle excitation levels observed during decreasing force (i.e., F Fown mode) may be considered the compensatory behavior of spinal motoneuronal activity to produce similar levels of muscle tension [35].

The significant correlation between the $\mathrm{tHb}$ and $\mathrm{TSI}_{\text {SLOPE }}$ during both the initial and terminal steady-state force production implies that the reduction in blood flow by the increased muscle tension is generally accompanied by the promotion of oxygen consumption compared to its supply, and vice versa [36,37]. Another interesting observation in the current results is that both the blood volume and oxygen demand index at the $\mathrm{MVC}_{50}$ did not have parallel changes in terms of the history of changes in force magnitudes (e.g., $\Delta \mathrm{F}_{10}$ and $\Delta \mathrm{F}_{20}$ ) over time, which was different from the result of the $i$ EMG. It is generally agreed that the IMP is modulated as the intensity of voluntary change of muscle contraction. In particular, the changes in $\mathrm{tHb}$ are indicative of the modulation in the IMP $[36,38,39]$. Thus, the increased IMP by voluntary muscle contraction is associated with a reduction in the intramuscular blood volume. The history-effect of the blood volume in the current study may be determined whether the amounts of reduction and promotion of the blood supply rely on the time-history of the outcome force or not. In this regard, the relatively large blood volume after $F_{D O W N}$ as compared to after $F_{U P}$ could be affected by the rise in energetic demands. The values of TSISLOPE were unidirectional changes for the $\mathrm{F}_{\mathrm{UP}}$ and $\mathrm{F}_{\mathrm{DOWN}}$ modes as well, both of which were far below the linear regression line with larger the desatura- 
tion rate in the $\mathrm{F}_{\text {DOWn }}$ mode (Fig. 2 and 3). The levels of oxygen deficit rise with the intensity of the initial stage of muscle contraction. The increment in the blood supply may be a compensatory mechanism for the deficiency of oxygen supply when the force demand is reduced from the high level of force. Thus, it is highly probable that the amount of oxygenation demand is partly constrained by the intensity of muscle contraction and its time-history regarding its direction of magnitude change.

\section{CONCLUSION}

The main goal of the current study was to gain insight into the history-dependence on muscle excitation and oxygenation. The main result supports the specific hypotheses by demonstrating the history-dependent changes in the properties of muscle excitation and oxygenation that were quantified by the EMG and NIRS measures. Notably, the EMG and NIRS measures at the same level force were varied depending on the time history of contraction. The phenomenon of non-uniform changes in the EMG and NIRS indices suggests that the absolute values of outcome or muscle forces may not be the sole component to specify the levels of muscle excitation and oxygenation or vice-versa. However, the current outcomes should be viewed as tentative due to a few limitations of the current study. The relatively small sample size and no measurement of other muscles of the quadriceps and hamstring group for the knee extension are apparent drawbacks; thus, future studies will have to examine whether the current conclusions would be reached when the coordinated contractions of a set of quadriceps and the antagonist muscle group are considered with relatively large sample size. Furthermore, it will be interesting research topics to identify if the history-dependence of the physiological demands for voluntary muscle force production is valid in which the length of the muscle is changed (e.g., isotonic contraction, isometric contraction with different joint configurations, etc.)

\section{ACKNOWLEDGMENT}

This work was supported in part by the Ministry of Science and the National Research Foundation of Korea (NRF-2019R1F1A1061871), the Creative-Pioneering Researchers Program through Seoul National University (SNU), and the Nano Material Technology Development Program through the National Research Foundation of Korea (NRF) funded by the Ministry of Science, ICT and Future Planning (No .2016M3A7B4910552).

\section{CONFLICT OF INTERES}

The authors declare that the research was conducted in the absence of any commercial or financial relationships that could be construed as a potential conflict of interest.

\section{AUTHOR CONTRIBUTION}

Conceptualization: J Park, J Song; Data curation: J Song, S Lee, J Lee, K Kim; Formal analysis: J Park, J Song; Methodology: J Song, S Lee, J Lee; Project administration: J Park; Visualization: K Kim, J Song; Writing-original draft: J Park, J Song; Writing-review \& editing: J Park.

\section{ORCID}

$\begin{array}{ll}\text { Junkyung Song } & \text { https://orcid.org/0000-0002-4399-9323 } \\ \text { Kitae Kim } & \text { https://orcid.org/0000-0002-7589-7487 } \\ \text { Sungjun Lee } & \text { https://orcid.org/0000-0002-4963-6419 } \\ \text { Jiseop Lee } & \text { https://orcid.org/0000-0003-4384-5145 } \\ \text { Jaebum Park } & \text { https://orcid.org/0000-0003-0156-5591 }\end{array}$

\section{REFERENCES}

1. Colier W, Meeuwsen I, Degens H, Oeseburg B. Determination of oxygen consumption in muscle during exercise using near infrared spectroscopy. Acta Anaesthesiol Scand. 1995;39:151-5.

2. Yamada E, Kusaka T, Miyamoto K, Tanaka S, Morita, S, et al. Muscle oxygenation, muscle force and electromyographic activity during isometric contraction. Isokinet Exerc Sci. 2003;11(4):213-8.

3. Skovereng K, Ettema G, van Beekvelt M. Local muscle oxygen consumption related to external and joint specific power. Hum Movement Sci. 2016;45:161-71.

4. Felici F, Quaresima V, Fattorini L, Sbriccoli P, Filligoi G, et al. Biceps brachii myoelectric and oxygenation changes during static and sinusoidal isometric exercises. J Electromyogr and Kinesiol. 2009;19(2):e1-e11.

5. Jones AM, Davies RC, Ferreira LF, Barstow TJ, Koga S, et al. Evaluation of the dynamics of muscle oxygenation by near-infrared-based tissue oximeters. J Appl Physiol. 2009;107(1):372-3.

6. Zahalak GI, Ma SP. Muscle activation and contraction: constitutive relations based directly on cross-bridge kinetics. J Biomech Eng. 1990; 112(1):52-62. 
7. Davies R, Kushmerick M, Larson R. ATP, activation, and the heat of shortening of muscle. Nature. 1967;214(5084):148-51.

8. Westerblad H, Bruton JD, Katz A. Skeletal muscle: energy metabolism, fiber types, fatigue and adaptability. Exp Cell Res. 2010;316(18):3093-9.

9. De Luca CJ. The use of surface electromyography in biomechanics. J Appl Biomech. 1997;13(2):135-63.

10. Lloyd AJ. Surface electromyography during sustained isometric contractions. J Appl Physiol. 1971;30(5):713-9.

11. Maton B, Petitjean M, Cnockaert J. Phonomyogram and electromyogram relationships with isometric force reinvestigated in man. Eur J Appl Physiol Occup Physiol. 1990;60(3):194-201.

12. Praagman M, Chadwick E, Van Der Helm F, Veeger H. The relationship between two different mechanical cost functions and muscle oxygen consumption. J Biomech. 2006;39(4):758-65.

13. Wigmore D, Propert K, Kent-Braun J. Blood flow does not limit skeletal muscle force production during incremental isometric contractions. Eur J Appl Physiol. 2006;96(4):370-8.

14. De Ruiter CJ, Goudsmit JF, Van Tricht JA, De Haan A. The isometric torque at which knee-extensor muscle reoxygenation stops. Med Sci Sports Exerc. 2007;39(3):443-53.

15. Abbott B, Aubert X. The force exerted by active striated muscle during and after change of length. J Physiol. 1952;117(1):77.

16. Morgan DL, Whitehead NP, Wise AK, Gregory JE, Proske U. Tension changes in the cat soleus muscle following slow stretch or shortening of the contracting muscle. J Physiol. 2000;522(3):503-13.

17. Herzog W. History dependence of skeletal muscle force production: Implications for movement control. Hum Movement Sci. 2004;23(5): 591-604.

18. Kostyukov A. Muscle hysteresis and movement control: a theoretical study. Neuroscience. 1998;83(1):303-20.

19. Partridge LD. Modifications of neural output signals by muscles: a frequency response study. J Appl Physiol. 1965;20(1):150-6.

20. Ter Haar Romeny B, Van Der Gon JD, Gielen C. Relation between location of a motor unit in the human biceps brachii and its critical firing levels for different tasks. Exp Neurol. 1984;85(3):631-50.

21. Alkner BA, Tesch PA, Berg HE. Quadriceps EMG/force relationship in knee extension and leg press. Med Sci Sports Exerc. 2000;32(2):459-63.

22. Jacobs R, Schenau GJV. Control of an external force in leg extensions in humans. J Physiol. 1992;457:611-26.

23. Lee J, Song J, Ahn J, Park J. The effect of short-term muscle vibration on knee joint torque and muscle firing patterns during a maximal vol- untary isometric contraction. KJSB. 2017;27(2):83-90.

24. Katsavelis D, Threlkeld AJ. Quantifying thigh muscle co-activation during isometric knee extension contractions: Within- and betweensession reliability. J Electromyogr and Kinesiol. 2014;24(4):502-7.

25. Dimitrova NA, Dimitrov GV. Interpretation of EMG changes with fatigue: facts, pitfalls, and fallacies. J Electromyogr and Kinesiol. 2003;13 (1):13-36.

26. Kocsis L, Herman P, Eke A. The modified Beer-Lambert law revisited. Phys Med Biol. 2006;51(5):N91.

27. Jones B, Dat M, Cooper CE. Underwater near-infrared spectroscopy measurements of muscle oxygenation: laboratory validation and preliminary observations in swimmers and triathletes. J Biomed Opt. 2014;19(12):127002.

28. Muthalib M, Millet GY, Quaresima V, Nosaka K. Reliability of nearinfrared spectroscopy for measuring biceps brachii oxygenation during sustained and repeated isometric contractions. J Biomed Opt. 2010;15(1):017008.

29. Kutner MH, Nachtsheim CJ, Neter J, Li W, Applied linear statistical models. Vol. 5: McGraw-Hill Irwin New York; 2005.

30. Rehorn MR, Schroer AK, Blemker SS. The passive properties of muscle fibers are velocity dependent. J Biomech. 2014;47(3):687-93.

31. Taylor DC, Dalton JR JD, Seaber AV, Garrett JR WE. Viscoelastic properties of muscle-tendon units: the biomechanical effects of stretching. Am J Sports Med. 1990;18(3):300-9.

32. Gorassini M, Yang JF, Siu M, Bennett DJ. Intrinsic activation of human motoneurons: possible contribution to motor unit excitation. J Neurophysiol. 2002;87(4):1850-8.

33. Romaiguere P, Vedel J, Pagni S. Comparison of fluctuations of motor unit recruitment and de-recruitment thresholds in man. Exp Brain Res. 1993;95(3):517-22.

34. Denier Van Der Gon J, ter Haar Romeny B, Van Zuylen E. Behaviour of motor units of human arm muscles: differences between slow isometric contraction and relaxation. J Physiol. 1985;359(1):107-18.

35. Kimura T, Yamanaka K, Nozaki D, Nakazawa K, Miyoshi T, et al. Hysteresis in corticospinal excitability during gradual muscle contraction and relaxation in humans. Exp Brain Res. 2003;152(1):123-32.

36. Sejersted O, Hargens A. Intramuscular pressures for monitoring different tasks and muscle conditions. Fatigue. 1995. Springer.

37. Taelman J, Vanderhaegen J, Robijns M, Naulaers G, Spaepen A, et al., Estimation of muscle fatigue using surface electromyography and near-infrared spectroscopy, in Oxygen Transport to Tissue XXXII, 
Springer 2011. p. 353-9.

38. Al-Mulla MR, Sepulveda F, Colley M. A review of non-invasive techniques to detect and predict localised muscle fatigue. Sensors. 2011; 11(4):3545-94.
39. Yoshitake Y, Ue H, Miyazaki M, Moritani T. Assessment of lower-back muscle fatigue using electromyography, mechanomyography, and near-infrared spectroscopy. Eur J Appl Physiol. 2001;84(3):174-9. 